\title{
Freehand vs. Micro Gestures in the Car: Driving Performance and User Experience
}

\author{
Renate Häuslschmid ${ }^{1,2}$, Benjamin Menrad ${ }^{1,3}$, Andreas Butz $^{2}$ \\ ${ }^{1}$ IAV GmbH, ${ }^{2}$ University of Munich (LMU), ${ }^{3}$ University of Regensburg
}

\begin{abstract}
Until now, freehand and micro gestures have only been investigated separately. We conducted a driving simulator study to investigate on the effects on the driving performance when controlling a music player and the user experience provided. Subjects felt that stimulation, control, popularity, and physicalform were addressed by both gesture types, but slightly better by freehand gestures. But micro gestures were rated notably higher regarding their perceived degree of autonomy. Regarding driving performance, deteriorations were found for both gesture types. Results indicate, freehand gestures impair lateral control while micro gestures delay steering.
\end{abstract}

\section{INTRODUCTION}

Currently, gestures are investigated intensively, especially in the context of driving. Previous studies can be categorized according to the different gesture types. By micro gestures (MG) we refer to small movements of one or more fingers from a fixed hand position, in contrast to freehand or mid-air gestures (FG), which are executed by moving the entire hand in space. Both gesture types have been found to be promising approaches for gesture interaction while driving [1,2].

In driving schools, drivers are taught to keep the view straight on the road and both hands on the wheel in a specific hand position in order to maintain good control of the vehicle [3, 4]. As the driver does not have to glance away from the road to control vehicle functions, gestures provide a high potential to increase safety. Though, to perform FG the driver still has to remove the right hand from the wheel. In contrast, MG allow the driver to leave both hands on the wheel and are not restricted to the right hand. Consequently, MG might be the overall safer approach [2].

Until now, FG and MG have only been investigated separately. Moreover, neither the impacts on driving performance nor the user experience provided by gestures have been studied in detail. The outcomes of the existing studies are hardly comparable and, therefore, nothing can be said about the superiority of one gesture type over the other in the context of driving. Nevertheless, most research focuses on FG while only few studies look at MG, but is this really justifiable?

\section{Gesture control}

As an experimental task we chose the control of a music player. Controlling music is a frequent tertiary task while driving and consists of six main user actions: Play and pause, next and previous song, increase and decrease volume. As gestures we chose motions which are easy to memorize and are applicable to both gesture types (see table 1). This guaranteed the gestures were

\footnotetext{
${ }^{1}$ renate.haeuslschmid@gmail.com

2 benjamin.menrad@iav.de

${ }^{3}$ butz@ifi.lmu.de
}

equally intuitive and comparable. To capture the gestures a leap motion sensor was used. It was either positioned behind or to the right of the steering wheel, as proposed in $[1,2]$.

\section{Table 1: The used gesture sets. Graphics are partially} obtained out of [5].

Gesture
Play/ Pause
An open palm with fingers
spread for playing/ pausing
the music.
Next/Previous
A swipe gesture to the
switch to the next/ previous
song.
Louder/Softer
A clockwise/ counterclock-
wise circle gesture to
control the volume.

\section{STUDY}

For our dual task driving simulator study we chose a between subjects design. As driving task, we chose the standardized Lane Change Task (LCT), provided by the used openDS driving simulator. As secondary task subjects had to control a music player. Each of the three baseline and two intervention phases was scheduled with 3 minutes and 18 randomized lane changes (LCs). Intervention sections additionally challenged the driver with 18 randomized gestures. As gesture triggers we decided to use audio commands to avoid a visual overload.

We recruited 24 participants; 12 for each subject group. Most subjects drove frequently. 16 participants (FG: 8; MG: 7) drove frequently with both hands on the steering wheel, whereas 9 subjects (FG: 4; MG: 5) often drove with one hand only.

Before the test started, participants completed a demographic questionnaire and were introduced to the music player and the gestures. The test procedure was split into a habituation and a testing phase. After the test participants completed the user experience questionnaire.

\section{Results And Discussion}

\subsection{Driving Performance}

Mean Deviation corresponds to the deviation from the actually driven to the optimal driving line. The results for mean deviation are depicted in figure 1. To analyze these values, we performed two separate repeated measure ANOVAs. For both gesture interaction types we found a significant increase in mean deviation from baseline to intervention with $\mathrm{p}=.0336\left(\mathrm{~F}_{1,11}=5.885\right)$ for $\mathrm{FG}$ and $\mathrm{p}=.0014\left(\mathrm{~F}_{1,11}=17.914\right)$ for $\mathrm{MG}$. We also performed a mixed repeated measures ANOVA to compare both gesture types directly. Here, we found that $\mathrm{MG}$ deteriorated mean deviation significantly more than $\mathrm{FG}\left(\mathrm{F}_{1,22}=4.321 ; \mathrm{p}=.0495\right)$. 


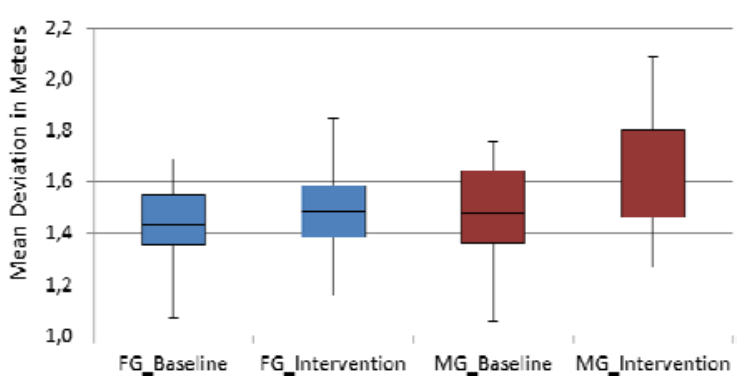

Figure 1: Deviation from optimal driving line (FG: blue; MG: red)

Reaction time represents the time between the appearance of the LC command and the driver's steering reaction. The measurements for reaction time as well as the times to complete a LC are presented in table 2 . We performed the same tests as for mean deviation and found significant impairment of reaction time for FG $\left(\mathrm{F}_{1,11}=6.533 ; \mathrm{p}=.0267\right)$ and $\mathrm{MG}\left(\mathrm{F}_{1,11}=14.011 ; \mathrm{p}=.0032\right)$. We did not find a significant difference between both gesture types.

Table 2: Time to react and to complete a lane change

\begin{tabular}{lcccc} 
Time & \multicolumn{2}{c}{ Freehand Gestures } & \multicolumn{2}{c}{ Micro Gestures } \\
& Baseline & Interven. & Baseline & Interven. \\
\hline Reaction & $621 \mathrm{~ms}$ & $695 \mathrm{~ms}$ & $656 \mathrm{~ms}$ & $773 \mathrm{~ms}$ \\
Completion & $2168 \mathrm{~ms}$ & $1986 \mathrm{~ms}$ & $2179 \mathrm{~ms}$ & $2192 \mathrm{~ms}$
\end{tabular}

Success rate presents the proportion of successfully performed LCs in relation to the maximum. A LC is successful when the driver maintained stable control and when it was performed within $5000 \mathrm{~ms}$. The results for the success rates are depicted in table 3. The ANOVA tests revealed a significant impact on the success rate for FG $\left(F_{1,11}=6.569 ; p=.0264\right)$ but not for $\mathrm{MG}$ or the comparison of both gesture types.

Table 3: Successfully performed lane changes

\begin{tabular}{lccc} 
Gesture Type & Baseline & Intervention & Difference \\
\hline Freehand G. & $87.7 \%$ & $75.0 \%$ & $-12.7 \%$ \\
Micro G. & $86.4 \%$ & $88.0 \%$ & $+1.5 \%$
\end{tabular}

Results for mean deviation, the primary measurement of the $\mathrm{LCT}$, indicate that FG deteriorate driving performance less than MG. Though, we think the low success rate and the slightly increased reaction time in interaction with the lowered completion time indicates that FG impede lateral control. In the beginning we claimed that removing one hand from the steering wheel is not the safest option. Though, performing MG while steering with the same hand does not seem to be safer. The conflict of steering (where the hand turns with the wheel) and the repositioning of the hand on the wheel to perform the gestures horizontally and within the recognition area most probably caused the deteriorations in mean deviation and reaction time. We assume subjects first finished the gesture and then finished the LC (mostly successful).

\subsection{User Experience}

Our questionnaire addressed the psychological needs stimulation, control, popularity, physical-form, and autonomy and contained few additional questions about the user's preferences.

Stimulation (FG: 4.0; MG: 3.9) Participants enjoyed performing gestures and found to be a fascinating experience.

Control (FG: 3.5; MG: 3.4) Subjects felt to be in control and to act fairly effective. They did not feel very safe and secure, but interaction was perceived as predictable and non-threatening.

Popularity (FG: 3.1; MG: 3.0) Subjects rated gesture control better than standard haptic buttons for music player control. Quite surprisingly, participants felt rather ashamed than proud being observed when using the gesture control.

Physical-form (FG: 3.3; MG: 3.0) Subjects felt not very active but comfortable in their body. Participants performing freehand gestures felt higher physical strain and more vital.

Autonomy (FG: 1.7; MG: 2.4) Participants stated, that they had little freedom of action and decision. They felt that they acted under pressure from the outside and were not able to behave according to their interests and values. A system that forced participants to change their habitual hand position was rated on average 0.5 points lower.

Both gesture types were appreciated by the participants. Subjects would use gesture interaction in their own car and spend $€ 50$ to $€ 900$ ( $€ 250$ on average for both types) for it. Whereas MG were rated notably higher in autonomy, FG were rated slightly better in stimulation, control, popularity, and physical-form.

\section{Conclusions}

In the recent gesture interaction research, micro gestures, as an alternative to freehand gestures, have been neglected. In this study freehand and micro gestures were directly compared regarding driving performance and user experience.

Generally, both gesture types provided good user experience. While participants rated gestures as very stimulating, they also pointed at a lack of autonomy. The psychological basic needs control, popularity and physical-form were rated quite neutral.

Both gesture types were found to impair driving performance, but differently. Results indicate freehand gestures lead to a bad lateral control and micro gestures cause delayed steering. Steering, and thereby turning the hand, requires a constant repositioning of the hand on the wheel to perform the gestures correctly. If this problem can be solved, micro gestures can support individual preferences, handedness, and hand positioning habits and provide a good alternative to FG.

After all, we did not identify one gesture type as generally superior in user experience and driving performance. In light of this study, we argue that the neglect of micro gestures in current research is not justifiable. We encourage further exploration and investigation of micro and freehand gestures. It is important to consider handedness as well as hand positioning and steering habits to see if the conflict of steering and gesturing can be mitigated. Such a study would benefit from not restricting micro gestures to one or the right hand.

\section{RefERENCES}

[1] S. Loehmann, M. Knobel, M. Lamara, and A. Butz. Culturally Independent Gestures for In-Car Interactions. Proceedings of INTERACT'13, (Cape Town, South Africa, 2013), pages 538-545. Springer Berlin Heidelberg, 2013.

[2] A. Mahr, C. Endres, C. Müller, and T. Schneeberger. Determining human-centered parameters of ergonomic micro-gesture interaction for drivers using the theater approach. Proceedings of Automotive User Interfaces and Interactive Vehicular Applications '11, (Salzburg, Austria, 2011), pages 151-158. ACM New York, 2011.

[3] Land Transport Safety Authority. Official New Zealand Road Code and Licence Guide for Car Drivers. DLM Campaign Ltd., 1999.

[4] D. Walton and J. A. Thomas. Naturalistic observations of driver hand position. in Lajunen, T., Özkan, T., Porter, B. E. Transportation Research Part F: Traffic Psychology and Behaviour, pages 229-238. Elsevier Ltd., 2005.

[5] T. Piumsomboon, A. Clark, M. Billinghurst, and A. Cockburn. Userdefined gestures for augmented reality. Proceedings of CHI EA'13 (Paris, France, 2013), pages 955-960. ACM New York, 2013. 